\title{
BMJ Open Protocol for a multicentre, prospective, population-based cohort study of variation in practice of cholecystectomy and surgical outcomes (The CholeS study)
}

Ravinder S Vohra, ${ }^{1,2,3}$ Philip Spreadborough, ${ }^{1}$ Marianne Johnstone, ${ }^{1}$ Paul Marriott, ${ }^{1}$ Aneel Bhangu, ${ }^{1,2}$ Derek Alderson, ${ }^{2,3}$ Dion G Morton, ${ }^{1,2}$ Ewen A Griffiths, ${ }^{3}$ On behalf of the West Midlands Research Collaborative

To cite: Vohra RS,

Spreadborough $P$, Johnstone $\mathrm{M}$, et al. Protocol for a multicentre, prospective, population-based cohort study of variation in practice of cholecystectomy and surgical outcomes (The CholeS study). BMJ Open 2015;5:e006399. doi:10.1136/bmjopen-2014006399

- Prepublication history for this paper is available online. To view these files please visit the journal online (http://dx.doi.org/10.1136/ bmjopen-2014-006399).

Received 17 August 2014 Revised 25 November 2014 Accepted 16 December 2014

CrossMark

\begin{abstract}
${ }^{1}$ West Midlands Research Collaborative, University of Birmingham, Birmingham, UK

${ }^{2}$ Academic Department of Surgery, University of Birmingham, Birmingham, UK

${ }^{3}$ Department of Upper GI Surgery, Queen Elizabeth Hospital, University Hospitals Birmingham NHS Foundation Trust, Birmingham, UK
\end{abstract}

Correspondence to Ewen A Griffiths; ewen.griffiths@uhb.nhs.uk

\section{ABSTRACT}

Introduction: Cholecystectomy is one of the most common general surgical operations performed. Despite level one evidence supporting the role of cholecystectomy in the management of specific gallbladder diseases, practice varies between surgeons and hospitals. It is unknown whether these variations account for the differences in surgical outcomes seen in population-level retrospective data sets. This study aims to investigate surgical outcomes following acute, elective and delayed cholecystectomies in a multicentre, contemporary, prospective, population-based cohort.

Methods and analysis: UK and Irish hospitals performing cholecystectomies will be recruited utilising trainee-led research collaboratives. Two months of consecutive, adult patient data will be included. The primary outcome measure of all-cause 30-day readmission rate will be used in this study. Thirty-day complication rates, bile leak rate, common bile duct injury, conversion to open surgery, duration of surgery and length of stay will be measured as secondary outcomes. Prospective data on over 8000 procedures is anticipated. Individual hospitals will be surveyed to determine local policies and service provision. Variations in outcomes will be investigated using regression modelling to adjust for confounders.

Ethics and dissemination: Research ethics approval is not required for this study and has been confirmed by the online National Research Ethics Service (NRES) decision tool. This novel study will investigate how hospital-level surgical provision can affect patient outcomes, using a cross-sectional methodology. The results are essential to inform commissioning groups and implement changes within the National Health Service (NHS). Dissemination of the study protocol is primarily through the trainee-led research collaboratives and the Association of Upper Gastrointestinal Surgeons (AUGIS). Individual centres will have access to their own results and the collective results of the study will be published in peer-reviewed journals and presented at relevant surgical conferences.

\section{Strengths and limitations of this study}

- A multicentre, prospective study, with independent validation of data.

- Capture of practice throughout the UK and Ireland.

- Data from all sizes of hospital and levels of specialist services.

- Inability to assess postoperative visits to general and family practitioners.

- Unable to capture the patients representing to other hospitals.

- Complications after 30 days not collected.

- Reason for acute surgery decision may not be fully elucidated.

\section{INTRODUCTION}

Cholecystectomy is one of the most commonly performed general surgical procedures in the UK. Approximately 66000 cholecystectomies were performed during the 2011-2012 financial year in England alone. ${ }^{1}$ The pathway for these patients can be divided into three distinct groups: (1) acute admission with biliary disease and the cholecystectomy performed during that acute admission (acute group); (2) planned elective admission for cholecystectomy referred by their family doctor and added to the routine surgical waiting list from the outpatient department only (elective group) and (3) all other planned cholecystectomies performed on an elective operating list, who have had a previous emergency surgical admission with gallbladder (GB)-related disease (delayed group). This is shown in figure 1 .

The management of GB disease and whether patients have acute, elective or delayed operations varies widely between surgeons and hospitals. ${ }^{2-4}$ Level one evidence 
Figure 1 The pathway for patients who undergo cholecystectomy. Three groups of patients will be assessed: (1) acute admission with biliary disease and the cholecystectomy performed during that acute admission (acute group); (2) planned elective admission for cholecystectomy referred by their family doctor and added to the routine surgical waiting list from the outpatient department only (elective group) and (3) all other planned cholecystectomies performed on an elective operating list, who have had a previous emergency surgical admission with gallbladder-related disease (delayed group).

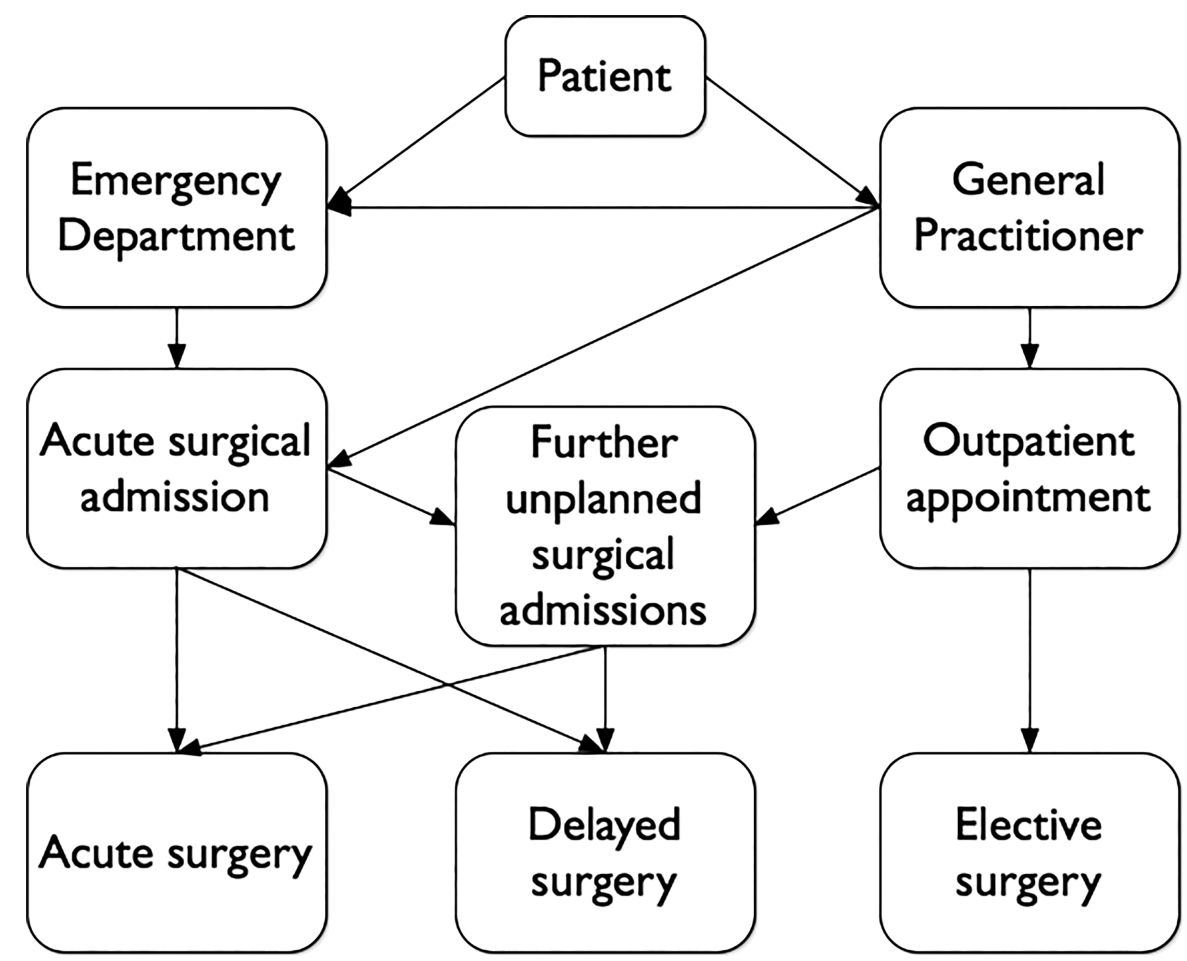

supports the role and safety of early or acute laparoscopic cholecystectomy in biliary colic, cholecystitis and gallstone pancreatitis. ${ }^{5-10}$ Population-level data from Hospital Episodes Statistics (HES) and a retrospective study from Scotland, both suggest however, that early or acute operations may be associated with poor surgical outcomes. $^{2} 3$ These studies also suggest that differences in outcomes may be linked to hospital size and volume. ${ }^{23}$

Different surgical outcomes are commonly used to measure quality of healthcare, such as readmissions, reoperations and mortality. In particular, reducing hospital readmissions following surgery can lower hospital costs and improve patient satisfaction. ${ }^{11}$ The causes of readmission after cholecystectomy have been poorly studied, but are likely to vary by hospital size, case volume and whether patients have acute, elective or delayed operations performed. ${ }^{12}$

Taken together, this raises the possibility that trial evidence of common surgical procedures is not generalisable to population level, non-trial cohorts. Furthermore, differences in patient outcomes may be linked to hospital service provision. These factors both have an important impact on patients and the National Health Service (NHS), especially as trial data is in part used to inform commissioning decisions. It is not clear currently, why such variations occur in the NHS, or what the impact of these variations is on surgical outcomes.

\section{METHODS AND ANALYSIS}

Primary aim

To measure the difference in all-cause 30-day readmissions following acute, delayed and elective cholecystectomies in a contemporary, population-based cohort.
Hypothesis

The 30-day readmission rate, following risk adjustment, should be equivalent in patients following elective cholecystectomies compared with acute and delayed procedures.

\section{Study design}

We plan to undertake a multicentre, contemporary, prospective, cohort audit which will be conducted through trainee-led research collaboratives as described previously. $^{13}$

\section{Setting}

This study can take place in any UK or Irish hospital performing acute, elective or delayed cholecystectomies. Each centre will contribute 2 months of consecutive patient data.

\section{Participants}

Inclusion criteria: All patients over the age of 18 years who are undergoing a cholecystectomy can be entered into this audit.

Exclusion criteria: Patients having a cholecystectomy for known GB cancer, or as a part of another surgical procedure for example, Whipple's procedure, bariatric, antireflux or transplant operations, will be excluded.

\section{Outcome measures}

The primary outcome measure is all-cause 30-day readmission rate, which is defined as any admission following discharge which requires an overnight stay. This standard and definition is based on the Royal College of Surgeons (RCS) and the Association of Upper Gastrointestinal Surgeons (AUGIS) of Great Britain and Ireland guidance 
Table 1 Secondary outcome measures

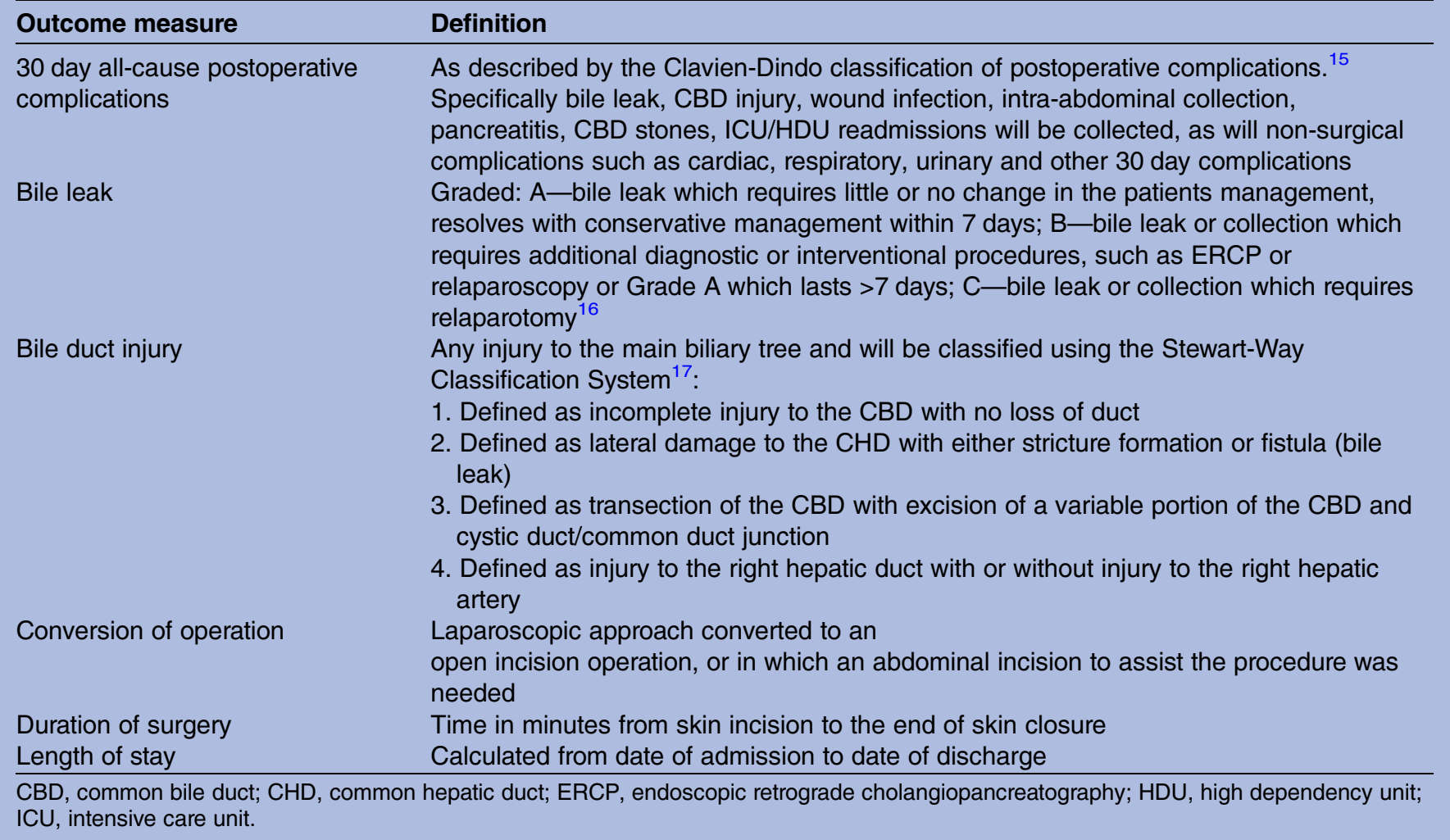

which states that the audit standard of less than $10 \%$ 30-day readmission rate should be reached by hospitals performing cholecystectomies. ${ }^{4}$ Secondary outcome measures are listed in table 1.

\section{Data collection}

Data will be collected in a standardised Microsoft Excel spreadsheet. It will be the responsibility of the local investigators to ensure that the data is password protected and held on local trust computer systems. Each trust/hospital site will need to identify locations where laparoscopic cholecystectomy are performed (main theatre, day-case unit, treatment centre) to ensure full capture of cases during the audit period. Patients will be identified on a daily basis from the elective operating lists and by on-call teams, at handovers, from on-call lists and from emergency theatre booking lists and logbooks. Operative data should be completed either by or with input from the operating surgeon or the assistant. All patients will be followed for 30 days following their operation. The hospital's electronic or paper records should be checked by the team to identify any readmissions or reattendances to the hospital's emergency department, surgical assessment unit or wards. Local arrangements may include:

- Reviewing the patient or patient's notes during admission to identify inpatient complications.

- Check the discharge summary or letter to check for any postoperative complications.

- Check for any outpatient attendances within 30 days of surgery.
- Check electronic or paper hospital records or handover lists for reattendances or readmissions. Check for any accident and emergency department reattendances.

- Review imaging reports or laboratory results to check for unplanned attendances which may have occurred.

\section{Data validation and management}

Following data collection, only data sets with $>95 \%$ data completeness will be accepted for pooled national analysis. The consultant surgeon principle investigator (PI) at selected sites will identify an independent assessor to validate all data points, with a target of $>98 \%$ accuracy. Overall, at least $5 \%$ of the data set will be independently validated. In addition, the independent assessor will be asked to examine operating theatre logbooks and trust data systems, to ensure case ascertainment. If the concordance between the total number of cases submitted to the trial management group is $<95 \%$, the hospital's data will be removed from the analysis.

A standardised Microsoft Excel spreadsheet (Excel 2007; Microsoft, Redmond, Washington, USA) with preset fields will be used to collect the data. Data protection regulations at each centre will be complied with. Patient identifiable data will not be transmitted to the trial management group. Data will be submitted centrally via a secure NHS email address with all patient identifiers removed. Patient anonymised data will be then be analysed and reported by the writing committee. Outcome data specific to each individual surgeon who participates will not be collected. Anonymised hospital data will be 
Table 2 Data fields

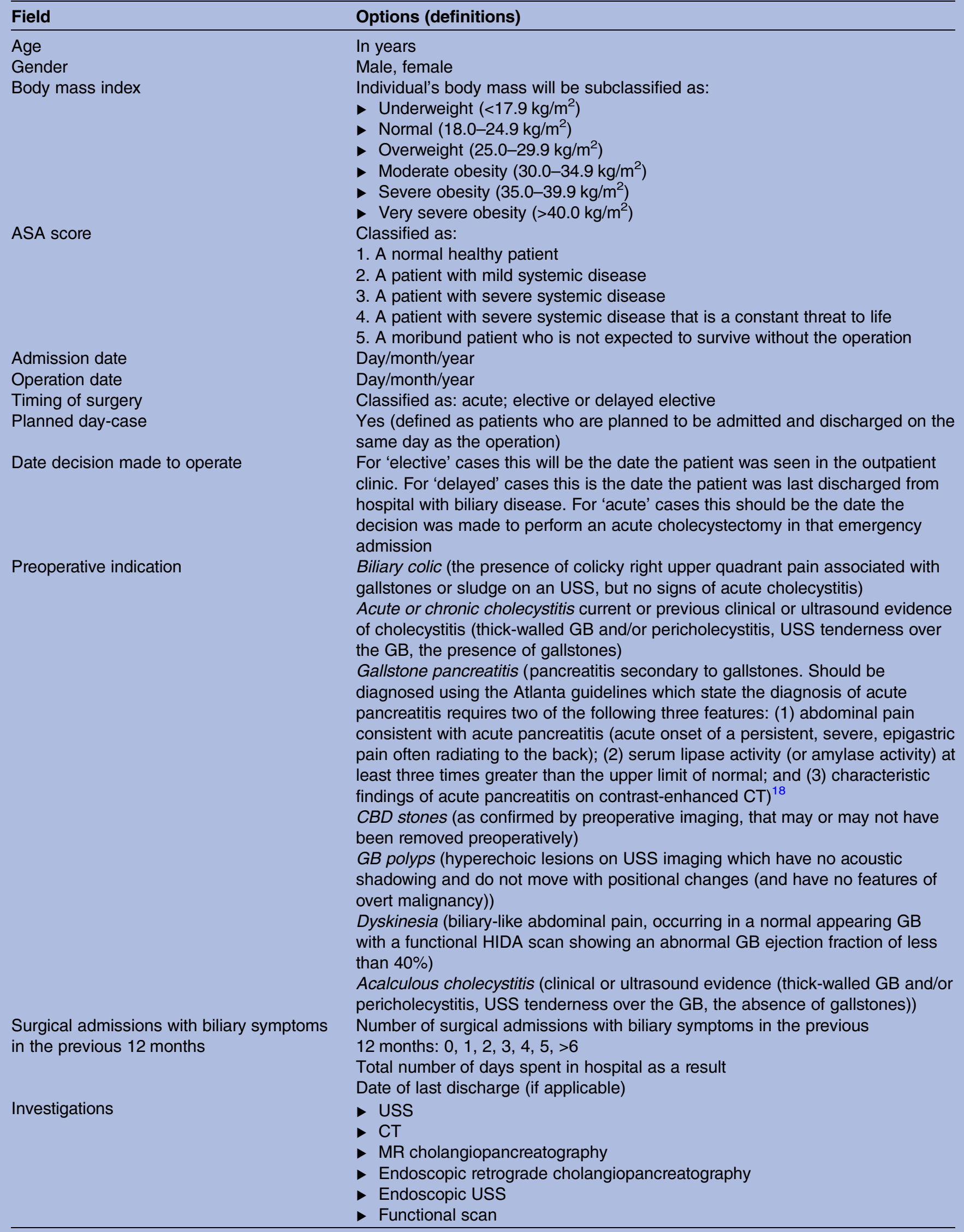


Table 2 Continued

\begin{tabular}{|c|c|}
\hline Field & Options (definitions) \\
\hline Seniority of surgeons & $\begin{array}{l}\text { <Specialty trainee (ST6) } \\
\text { ST6 or above or staff grade } \\
\text { Consultant }\end{array}$ \\
\hline Perioperative antibiotics & Yes/no \\
\hline Method of operation & $\begin{array}{l}\text { Laparoscopic } \\
\text { Laparoscopic converted to open } \\
\text { Open cholecystectomy } \\
\text { SILS }\end{array}$ \\
\hline Degree of difficulty & $\begin{array}{l}\text { Nassar scale of difficulty for cholecystectomy graded } 1,2,3,4^{19} \\
\text { Grade I Floppy, non-adherent gallbladder. Clear, thin cystic pedicle. Simple } \\
\text { adhesions to neck and Hartmann's pouch only } \\
\text { Grade II Mucocele; packed with stones gallbladder. Fat-laden cystic pedicle. } \\
\text { Simple adhesions, up to the body of gallbladder } \\
\text { Grade III Deep fossa; acute cholecystitis; contracted, fibrous } \\
\text { Hartmann's pouch adherent to CBD or with stone impaction. } \\
\text { Abnormal anatomy; cystic duct short, dilated or obscured. } \\
\text { Dense adhesions, up to the fundus; involving hepatic flexure or duodenum } \\
\text { Grade IV Completely obscured gallbladder; empyema/gangrene } \\
\text { Or mass. Impossible to clarify cystic pedicle. Dense, fibrous adhesions } \\
\text { wrapping the GB. Duodenum or hepatic flexure difficult to separate }\end{array}$ \\
\hline Intraoperative complications & $\begin{array}{l}\text { Bile spilt (intra-abdominal spillage of bile during the procedure, including } \\
\text { when removing the GB from the abdominal cavity) } \\
\text { Stones spilt (intra-abdominal spillage of stones during the procedure, } \\
\text { including as removing the GB from the abdominal cavity) } \\
\text { Bleeding (requiring haemostatic agents (eg, Surgicel, Fibrillar, etc), extra } \\
\text { clips, suturing or conversion to open procedure) } \\
\text { CBD injury (will be defined as any injury to the main biliary tree and will be } \\
\text { classified using the Stewart-Way Classification System }(1,2,3,4) \text { ) }\end{array}$ \\
\hline Intraoperative cholangiography & $\begin{array}{l}\text { Planned (defined as the decision to perform a cholangiogram before the } \\
\text { operation starts; eg, due to surgeon preference or to assess for CBD stones) } \\
\text { Unplanned (defined as any other reason where a cholangiogram was } \\
\text { performed but was not anticipated at the start of the operation; eg, to assess } \\
\text { for unclear anatomy or to assess for potential CBD injury) }\end{array}$ \\
\hline CBD exploration & Yes/no \\
\hline Abdominal drain & Yes/no \\
\hline Date of discharge & Day/month/year \\
\hline All-cause 30-day A\&E attendance & Yes/no \\
\hline All 30-day reinterventions and reimaging & Yes/no \\
\hline 30-day mortality & Yes/no \\
\hline
\end{tabular}

A\&E, accident and emergency department; ASA, American Society of Anesthesiologist; CBD, common bile duct; GB, gallbladder;

HIDA, hepatobiliary iminodiacetic acid; SILS, single-incision laparoscopic surgery; USS, ultrasound scan.

compared; individual surgeons, hospitals or NHS Trusts will not be identified. The required anonymous data fields are shown in table 2. Individual centres will be surveyed at the end of the audit period to determine local policies and service provision (table 3). Anonymous patient-level data will be linked to the results of the hospital service survey to allow detailed analysis of these variables and patient outcome.

\section{Anticipated recruitment}

On the basis of cholecystectomy-specific HES data from England, 66000 procedures were coded during the 2011-2012 financial year. ${ }^{1}$ If there is a uniform distribution of procedures performed between each of the acute care trusts and all hospitals in the UK and Ireland participate, data on 11000 procedures could be potentially gathered prospectively. Minimum expected recruitment would be approximately 1500 procedures from 20 centres from the West Midlands.

\section{Study timeline}

Data collection and analysis will be performed using the following timelines:

- 4 November 2013-11 December 2013-Pilot study period.

- 1 March 2014-1 May 2014-Main study data collection period.

- 1 June 2014-Main study 30-day follow-up period ends. 
Table 3 Hospital-level variables

\begin{tabular}{|c|c|}
\hline Field & Option (definition) \\
\hline Location & $\begin{array}{l}\text { England; Scotland; Wales; Northern Ireland; } \\
\text { Republic of Ireland }\end{array}$ \\
\hline University hospital & Yes/no \\
\hline Total number of beds & $<100 ; 100-500 ; 500-1000 ;>1000$ \\
\hline Tertiary HPB services & Yes/no \\
\hline ERCP services & Yes/no \\
\hline Acute admissions & Yes/no \\
\hline Number of consultants on the general surgery on-call rota & Number \\
\hline Consultant specialties involved in performing cholecystectomies & $\begin{array}{l}\text { Oesophagogastric } \\
\text { Hepatobiliary } \\
\text { Colorectal } \\
\text { Breast } \\
\text { Vascular } \\
\text { Endocrine } \\
\text { General } \\
\text { Transplant } \\
\text { Other }\end{array}$ \\
\hline Number of consultant surgeons offering cholecystectomy & Number \\
\hline Number of consultant surgeons offering acute/emergency cholecystectomy & Number \\
\hline Number of consultant surgeons offering laparoscopic CBD exploration & Number \\
\hline Does your hospital offer dedicated 'hot' gallbladder theatre lists & Yes/no \\
\hline Performing an intraoperative cholangiography & Yes/no \\
\hline \multirow[t]{6}{*}{ Additional hospital services available } & MRCP \\
\hline & Endoscopic USS \\
\hline & Functional scans \\
\hline & Radiological drainage \\
\hline & Percutaneous transhepatic cholangiograms \\
\hline & Radiological cholecystostomy \\
\hline
\end{tabular}

CBD, common bile duct; ERCP, endoscopic retrograde cholangiopancreatography; HPB, hepato-pancreato-biliary; MRCP, MR cholangiopancreatography; USS, ultrasound scan.

- 31 August 2014-Central data submission anticipated being complete.

- 20 December 2014-Independent data validation anticipated being complete.

- 28 February 2015-Initial data analysis anticipated being complete.

\section{Statistical analysis}

The report of this study will be prepared in accordance to guidelines set by the STROBE (Strengthening the Reporting of Observational Studies in Epidemiology) statement for observational studies. ${ }^{14}$ Data will be collected and analysed in clinically relevant categories, and $\chi^{2}$ tests used to detect differences between groups. Missing data will be analysed and multiple imputation used if required.

Binary logistic regression modelling will be used to adjust the influence of timing of surgery for key confounding variables. Data will be adjusted for patient (age, gender, American Society of Anesthesiologist (ASA) score and body mass index), disease (indication, ultrasound scan findings, the need of other imaging and endoscopic retrograde cholangiopancreatography (ERCP)), surgeon (consultant present, specialty and number of surgeons performing cholecystectomy) and hospital (number of hospital beds, university hospital, tertiary hepato- pancreato-biliary services, acute admissions, ERCP services and 'Hot' GB lists) factors. Multivariate models will be built to produce ORs to account for the impact of predictive variables when assessing outcomes. The OR represents the odds of all-cause 30-day readmission occurring, comparing the experimental groups (acute and delayed) versus the reference group (elective). A second model will compare acute cholecystectomy against a reference of delayed cholecystectomy. Variable selection including hospital-level data will be based on those which are statistically significant at univariate analysis, and those which are clinically significant, but not statistically. Depending on data requirements, models will be developed using fixed-entry binary logistic regression, multilevel modelling and multiply imputed data sets, to compare outputs based on statistical technique.

The main strength of this project is the multicentre, prospective, contemporary methodology with independent validation of the data. This will give high quality, validated data on cholecystectomy provision and outcome throughout the UK and Ireland from a wide range of hospital types.

Limitations include the inability to assess postoperative visits to the general practitioner. In addition, a minority of patients will re-present to other hospitals with complications following surgery. However, during the audit 
period, teams will also document the absolute number of patients readmitted with complications from cholecystectomy performed at other hospitals. Our study uses the standard 30 day follow-up period as this is the international standard and allows comparison with other studies. However, complications which may occur after 30 days, such as retained common bile duct stones or late biliary strictures will not be reported. In the acute group, it may not be possible to fully elucidate whether the decision to operate was due to hospital policies, surgeon preference or patient-related factors. However, we will collect data on patient, disease, surgeon and hospital factors to assess which is associated with acute cholecystectomy.

\section{DISCUSSION}

Variations in outcomes following common surgical procedures such as cholecystectomy are a concern for the NHS. This paper describes the protocol for a novel study to investigate how hospital-level surgical provision can affect patient outcomes using cross-sectional methodology. The results of this audit are essential to inform commissioning groups and implement changes within the NHS. Furthermore, the development of consultant PIs across the UK enables a network of new investigators who can be approached to collaborate on further studies.

\section{ETHICS AND DISSEMINATION}

The proposed study will not affect clinical care and compares an outcome to a defined audit standard. Research ethics approval is not required for this study and this has been confirmed by the use of the online National Research Ethics Service (NRES) decision tool (http:// www.hra-decisiontools.org.uk/research/). This has been further supported by written confirmation and advice from the Research and Development Director at University Hospitals Birmingham NHS Foundation Trust, UK. The study will be registered as a clinical audit or service evaluation at each participating hospital. Patient consent is not deemed necessary and inclusion in the study will not incur any risk to individual patients.

The generic collaborative methodology underlying protocol dissemination and collaborator recruitment has been described previously. ${ }^{13}$ The protocol will be disseminated primarily through the trainee-led research collaboratives (http://www.asit.org/resources/ collaboratives), and members of the Association of Surgeons in Training (ASIT; http://www.asit.org), AUGIS (http://www.augist.org) and the Schoolofsurgery.org (http://www.schoolofsurgery.org). A consultant surgeon PI will be designated at each hospital to facilitate coordination of the study. The protocol document and data collection tools will be made available online (http://www. choles-study.org). Individual centres will have access to their own results and the collective results of the study will be published in peer-reviewed journals and presented at relevant surgical conferences. These results can then be used to inform commissioning and implement changes within the NHS.

Contributors RSV and EAG contributed in the conception, design, writing and editing of the protocol. $A B, M J, P M, P S, D G M$ and DA contributed in the design and writing of the protocol. $A B$ and RSV participated in the statistical analysis. EAG is the guarantor. All authors read and approved the final manuscript.

\section{Competing interests None.}

Ethics approval NRES and Research and Development Director at University Hospitals Birmingham NHS Foundation Trust, UK.

Provenance and peer review Not commissioned; externally peer reviewed.

Data sharing statement It is the intention of the study group, that once the study is completed, and the research articles stemming from the study have been published, that any remaining data that is fully anonymised and not sensitive, be made open access.

Open Access This is an Open Access article distributed in accordance with the Creative Commons Attribution Non Commercial (CC BY-NC 4.0) license, which permits others to distribute, remix, adapt, build upon this work noncommercially, and license their derivative works on different terms, provided the original work is properly cited and the use is non-commercial. See: http:// creativecommons.org/licenses/by-nc/4.0/

\section{REFERENCES}

1. Hospital Episode Statistics. Total procedures and interventions. 2011-2012. http://www.hesonline.nhs.uk/Ease/servlet/ ContentServer?sitelD=1937\&categoryID=210 (accessed 3 Jul 2013).

2. Sinha S, Hofman D, Stoker DL, et al. Epidemiological study of provision of cholecystectomy in England from 2000 to 2009: retrospective analysis of Hospital Episode Statistics. Surg Endosc 2013;27:162-75.

3. Harrison EM, O'Neill S, Meurs TS, et al. Hospital volume and patient outcomes after cholecystectomy in Scotland: retrospective, national population based study. BMJ 2012;344:e3330.

4. AUGIS. 2013 Commissioning Guide: gallstone disease. http://www. rcseng.ac.uk/healthcare-bodies/docs/published-guides/gallstones (accessed 3 Jul 2013).

5. Macafee DA, Humes DJ, Bouliotis G, et al. Prospective randomized trial using cost-utility analysis of early versus delayed laparoscopic cholecystectomy for acute gallbladder disease. Br J Surg 2009;96:1031-40.

6. Gurusamy KS, Koti R, Fusai G, et al. Early versus delayed laparoscopic cholecystectomy for uncomplicated biliary colic. Cochrane Database Syst Rev 2013;6:CD007196.

7. Gurusamy K, Samraj K, Gluud C, et al. Meta-analysis of randomized controlled trials on the safety and effectiveness of early versus delayed laparoscopic cholecystectomy for acute cholecystitis. $\mathrm{Br} \mathrm{J}$ Surg 2010;97:624.

8. Siddiqui T, MacDonald A, Chong PS, et al. Early versus delayed laparoscopic cholecystectomy for acute cholecystitis: a meta-analysis of randomized clinical trials. Am J Surg 2008;195:40-7.

9. Falor $A E$, de Virgilio $C$, Stabile BE, et al. Early laparoscopic cholecystectomy for mild gallstone pancreatitis: time for a paradigm shift. Arch Surg 2012;147:1031-5.

10. UK Working Party on Acute Pancreatitis. UK guidelines for the management of acute pancreatitis. Gut 2005;54(Suppl III):iii1-9.

11. Tsai TC, Joynt KE, Orav J, et al. Variation in surgical-readmission rates and quality of hospital care. $N$ Engl $J$ Med 2013;369:1134-42.

12. Morris MS, Deierhoi RJ, Richman JS, et al. The relationship between timing of surgical complications and hospital readmission. JAMA Surg 2014;149:348-54.

13. Bhangu $A$, Kolias AG, Pinkney $T$, et al. Surgical research collaboratives in the UK. Lancet 2013;382:1091-2.

14. von Elm E, Altman DG, Egger M, et al. The Strengthening the Reporting of Observational Studies in Epidemiology (STROBE) statement: guidelines for reporting observational studies. Lancet 2007;370:1453-7.

15. Clavien PA, Barkun J, de Oliveira ML, et al. The Clavien-Dindo classification of surgical complications: five-year experience. Ann Surg 2009;250:187-96. 
16. Koch M, Garden OJ, Padbury R, et al. Bile leakage after hepatobiliary and pancreatic surgery: a definition and grading of severity by the International Study Group of Liver Surgery. Surgery 2011;149:680-8.

17. Way LW, Stewart L, Gantert W, et al. Causes and prevention of laparoscopic bile duct injuries: analysis of 252 cases from a human factors and cognitive psychology perspective. Ann Surg 2003;237:460-9.
18. Banks PA, Bollen TL, Dervenis C, et al; Acute Pancreatitis Classification Working Group. Classification of acute pancreatitis-2012: revision of the Atlanta classification and definitions by international consensus. Gut 2013;62:102-11.

19. Nassar AH, Ashkar KA, Mohamed AY, et al. Is laparoscopic cholecystectomy possible without video technology? Minim Invasive Ther Allied Technol 1995;4:63-5. 